\title{
Some Nonexistence and Asymptotic Existence Results for Weighing Matrices
}

\author{
Ebrahim Ghaderpour \\ Lassonde School of Engineering, York University, Toronto, ON, Canada M3J 1P3 \\ Correspondence should be addressed to Ebrahim Ghaderpour; ebig2@yorku.ca
}

Received 4 January 2016; Accepted 18 February 2016

Academic Editor: Christos Koukouvinos

Copyright ( 2016 Ebrahim Ghaderpour. This is an open access article distributed under the Creative Commons Attribution License, which permits unrestricted use, distribution, and reproduction in any medium, provided the original work is properly cited.

Orthogonal designs and weighing matrices have many applications in areas such as coding theory, cryptography, wireless networking, and communication. In this paper, we first show that if positive integer $k$ cannot be written as the sum of three integer squares, then there does not exist any skew-symmetric weighing matrix of order $4 n$ and weight $k$, where $n$ is an odd positive integer. Then we show that, for any square $k$, there is an integer $N(k)$ such that, for each $n \geq N(k)$, there is a symmetric weighing matrix of order $n$ and weight $k$. Moreover, we improve some of the asymptotic existence results for weighing matrices obtained by Eades, Geramita, and Seberry.

\section{Introduction}

An orthogonal design (OD) [1, Chapter 1] of order $n$ and type $\left(s_{1}, \ldots, s_{\ell}\right)$, denoted as $\operatorname{OD}\left(n ; s_{1}, \ldots, s_{\ell}\right)$, is a square matrix $X$ of order $n$ with entries from $\left\{0, \pm x_{1}, \ldots, \pm x_{\ell}\right\}$, where $x_{j}$ 's are commuting variables, that satisfies

$$
X X^{T}=\left(\sum_{j=1}^{\ell} s_{j} x_{j}^{2}\right) I_{n},
$$

where $X^{T}$ is the transpose of $X$ and $I_{n}$ is the identity matrix of order $n$. An OD with no zero entry is called a full OD. Equating all variables to 1 in any full OD results in a Hadamard matrix. Equating all variables to 1 in any OD of order $n$ results in a weighing matrix, denoted as $W(n, k)$, where $k$ is the weight that is the number of nonzero entries in each row (column) of the weighing matrix.

The Kronecker product of two matrices $A=\left[a_{i j}\right]$ and $B$ of orders $m \times n$ and $r \times s$, respectively, is denoted by $A \otimes B$, and it is the matrix of order $m r \times n s$ defined by

$$
A \otimes B=\left[\begin{array}{cccc}
a_{11} B & a_{12} B & \cdots & a_{1 n} B \\
a_{21} B & a_{22} B & \cdots & a_{2 n} B \\
\vdots & \vdots & & \vdots \\
a_{m 1} B & a_{m 2} B & \cdots & a_{m n} B
\end{array}\right] .
$$

The direct sum of $A$ and $B$ is denoted by $A \oplus B$, and it is the matrix of order $(m+r) \times(n+s)$ which is defined as follows:

$$
A \oplus B=\left[\begin{array}{ll}
A & 0 \\
0 & B
\end{array}\right],
$$

where 0 represents a zero matrix of appropriate dimension.

Let $A=\left(a_{1}, \ldots, a_{n}\right)$ and $D=\left(d_{1}, \ldots, d_{n}\right)$, where $a_{i}$ 's and $b_{i}$ 's belong to a commutative ring (e.g., $\left.\mathbb{R}\right)$. Square matrix $C=$ $\left[c_{i j}\right]$ of order $n$ is called circulant if $c_{i j}=a_{j-i+1}$, where $j-i$ is reduced modulo $n$. Square matrix $B=\left[b_{i j}\right]$ of order $n$ is called back-circulant if $b_{i j}=d_{i+j-1}$, where $i+j-2$ is reduced modulo $n$. We have $B C^{T}=C B^{T}$ and $B=B^{T}$; that is, any back-circulant matrix is symmetric. Let $R=\left[r_{i j}\right]$ be a square matrix of order $n$, where $r_{i j}=1$ if $i+j=1$ modulo $n$ and $r_{i j}=0$ otherwise. Matrix $R$ is called back-diagonal matrix. It is not hard to see that matrix $C R$ is back-circulant and so symmetric (cf. [1, Chapter 4]).

A rational family of order $n$ and type $\left(s_{1}, \ldots, s_{k}\right)$, where $s_{i}$ 's are positive rational numbers, is a collection of $k$ rational matrices of order $n, A_{1}, \ldots, A_{k}$, that satisfy $A_{i} A_{i}^{T}=s_{i} I_{n}$ $(1 \leq i \leq k)$ and $A_{i} A_{j}^{T}=-A_{j} A_{i}^{T},(1 \leq i \neq j \leq k)$. $A$ is a skew-symmetric matrix if $A^{T}=-A$. Two matrices of the same dimension are disjoint if their entrywise multiplication is a zero matrix $[1$, Chapters 1,2$]$. 
Geramita and Seberry [1, Chapter 7] showed some existence results for weighing matrices. They showed that when the orders of ODs and weighing matrices are much larger than the number of nonzero entries in each row, the necessary conditions for existence of ODs and weighing matrices are also sufficient. In this paper, we show some nonexistence results on weighing matrices and some asymptotic results for existence of weighing matrices.

\section{Nonexistence Results for Weighing Matrices}

In this section, we show some nonexistence results for weighing matrices. The results are summarized in Theorems 4 and 5 (known) and Theorem 10.

Lemma 1 (e.g., [2, Chapter 2]). The eigenvalues of a symmetric matrix with real entries are real.

Lemma 2 (e.g., [2, Chapter 2]). The eigenvalues of a skewsymmetric matrix with real entries are of the form $\pm i b$, where $b$ is a real number.

Lemma 3 (e.g., [1]). The absolute values of the eigenvalues of a weighing matrix $W(n, k)$ are $\sqrt{k}$.

Theorem 4 (e.g., [3]). There does not exist any symmetric weighing matrix with zero diagonal of odd order.

Proof. Suppose that $W=W(n, k), n$ odd, is a symmetric weighing matrix with zero diagonal. From Linear Algebra, $\operatorname{tr}(W)=\sum_{t=1}^{n} \lambda_{t}$, where $\lambda_{t}$ 's are eigenvalues of $W$. By Lemmas 1 and 3 , since $\lambda_{t}= \pm \sqrt{k}$,

$$
\operatorname{tr}(W)=\sum_{t=1}^{n} \lambda_{t}=c \sqrt{k} .
$$

Since $n$ is odd, $c$ must be odd and therefore nonzero, but, by assumption, $\operatorname{tr}(W)=0$, which is a contradiction.

Theorem 5 (e.g., [3]). There is no skew-symmetric weighing matrix of odd order.

Proof. Assume that $W=W(n, k)$ is a skew-symmetric weighing matrix of odd order. From Lemmas 2 and 3, eigenvalues of $W$ are in form $\pm i \sqrt{k}$. Therefore,

$$
\operatorname{tr}(W)=\sum_{t=1}^{n} \lambda_{t}=c i \sqrt{k} .
$$

Since $n$ is odd, $c$ must be odd and so nonzero, but since $W$ is a skew-symmetric matrix, $\operatorname{tr}(W)=0$ which is a contradiction.

Next, we show that if $n$ is any odd number and $k$ cannot be written as the sum of three integer squares, then there is no skew-symmetric weighing matrix $W(4 n, k)$. To do so, we first bring the following well known results.

Lemma 6 (e.g., [4]). A positive integer can be written as the sum of three integer squares if and only if it is not of the form $4^{\ell}(8 k+7)$, where $\ell, k \geq 0$.
The following lemma is a useful result that can be concluded from Lemma 6, and, for the sake of completion, we bring its proof.

Lemma 7 (e.g., [4]). A positive integer is the sum of three rational squares if and only if it is the sum of three integer squares.

Proof. Suppose that a positive integer $n$ is the sum of three rational squares. Reducing the three rational numbers to the same denominator, one may write

$$
m^{2} n=\alpha^{2}+\beta^{2}+\gamma^{2},
$$

where $\alpha, \beta$, and $\gamma$ are integers. Suppose that $n$ cannot be written as the sum of three integer squares. From Lemma 6 , there exist nonnegative integers $k$, $\ell$ such that $n=4^{\ell}(8 k+7)$. One may write $m$ as $2^{r}(2 s+1)$, for some nonnegative integers $r, s$. Thus, $m^{2}=4^{r}\left(4\left(s^{2}+s\right)+1\right)=4^{r}(8 b+1)$, where $b=\left(s^{2}+s\right) / 2$ is a nonnegative integer, and so

$$
m^{2} n=4^{r+\ell}(8 k+7)(8 b+1)=4^{r+\ell}(8 c+7),
$$

where $c=8 k b+k+7 b$. This is a contradiction because, by Lemma $6, m^{2} n$ cannot be written as the sum of three integer squares, whereas by assumption $m^{2} n=\alpha^{2}+\beta^{2}+\gamma^{2}$. Therefore, the result follows.

Lemma 8 (Shapiro [5]). There is a rational family in order $n=$ $2^{m} t, t$ odd, of type $\left(s_{1}, \ldots, s_{k}\right)$ if and only if there is a rational family of the same type in order $2^{m}$.

Lemma 9 (Geramita and Seberry [1]). A necessary and sufficient condition that there is a rational family of type $[1, k]$ in order 4 is that $k$ is a sum of three rational squares.

Proof. Suppose that $\{A, B\}$ is a rational family of type $[1, k]$ in order 4. Then $\left\{I=A^{T} A, D=A^{T} B\right\}$ is also a rational family of the same type and order. Thus $D=-D^{T}$ and $D D^{T}=k I$. Since $D$ is a skew-symmetric matrix, the diagonal of $D$ is zero, so $k$ is a sum of three rational squares.

Now let $k=a^{2}+b^{2}+c^{2}$, where $a, b$, and $c$ are rational numbers. If we let

$$
D=\left[\begin{array}{cccc}
0 & a & b & c \\
-a & 0 & -c & b \\
-b & c & 0 & -a \\
-c & -b & a & 0
\end{array}\right],
$$

then $\{I, D\}$ is a rational family of type $[1, k]$ and order 4 .

We use Lemmas 7, 8, and 9 to prove the following nonexistence result.

Theorem 10. Suppose that positive integer $k$ cannot be written as the sum of three integer squares. Then there does not exist skew-symmetric $W(4 n, k)$, for any odd number $n$.

Proof. If there is skew-symmetric $W=W(4 n, k)$ for some odd number $n$, then $\left\{I_{4 n}, W\right\}$ is a rational family of type $[1, k]$ 
and order $4 n$. Thus, by Lemma 8 , there is a rational family of type $[1, k]$ and order 4 . Lemmas 7 and 9 imply that $k$ must be the sum of three integer squares.

\section{Asymptotic Existence Results of Weighing Matrices}

In this section, we provide some asymptotic results for existence of weighing matrices. These results are summarized in Theorem 21 and Theorems 22 and 25 (for ODs) that use different methodologies to improve the known results shown by Geramita and Seberry [1, Chapter 7].

Lemma 11 (e.g., [1]). A necessary and sufficient condition that there exists $O D\left(n ; u_{1}, \ldots, u_{k}\right)$ is that there exists a family $\left\{A_{1}, \ldots, A_{k}\right\}$ of pairwise disjoint square matrices of order $n$ with entries from $\{0, \pm 1\}$ satisfying

(i) $A_{i}$ is a $W\left(n, u_{i}\right), 1 \leq i \leq k$,

(ii) $A_{i} A_{j}^{T}=-A_{j} A_{i}^{T}, 1 \leq i \neq j \leq k$.

The following lemma, due to Sylvester, is known, and we bring its proof.

Lemma 12 (see [6]). Let $x$ and $y$ be two relatively prime positive integers. Then every integer $N \geq x y$ can be written in the form $a x+b y$, where $a$ and $b$ are nonnegative integers.

Proof. Let $N$ be an integer greater than or equal to $x y$. Since $x$ and $y$ are relatively prime, there are integers $c$ and $d$ such that $c x+d y=N$ (see [4]). So,

$$
(c+j y) x+(d-j x) y=N,
$$

where $j \in \mathbb{Z}$. One can choose $j$ such that $0 \leq c+j y \leq y-1$. For such $j$, we let $a=c+j y$ and $b=d-j x$. The condition $N \geq x y$ implies that $b$ must be positive.

The following lemma shows how to construct ODs of higher orders by using two ODs of the same types but different orders. The first part of the lemma is known (cf. [1, Lemma 7.22]).

Lemma 13. Suppose that there are $A=O D\left(n_{1} ; u_{1}, \ldots, u_{m}\right)$ and $B=O D\left(n_{2} ; u_{1}, \ldots, u_{m}\right)$. Let $h=\operatorname{gcd}\left(n_{1}, n_{2}\right)$. Then there is an integer $N$ such that, for each $t \geq N$, there is $O D\left(h t ; u_{1}, \ldots, u_{m}\right)$. Moreover, if $A$ and $B$ are symmetric, then there is an integer $N$ such that, for each $t \geq N$, there is symmetric $O D\left(h t ; u_{1}, \ldots, u_{m}\right)$.

Proof. Let $x=n_{1} / h$ and $y=n_{2} / h$. Then $x$ and $y$ are relatively prime. Let $N=x y$, and let $t$ be a positive integer $\geq N$. By Lemma 12, there are nonnegative integers $a$ and $b$ such that $t=a x+b y$. Since there exist $\operatorname{OD}\left(n_{1} ; u_{1}, \ldots, u_{m}\right)$ and $\mathrm{OD}\left(n_{2} ; u_{1}, \ldots, u_{m}\right)$, there are families $\left\{A_{1}, \ldots, A_{m}\right\}$ of order $n_{1}$ and $\left\{B_{1}, \ldots, B_{m}\right\}$ of order $n_{2}$ satisfying the conditions in Lemma 11. We define the family

$$
S=\left\{\left(I_{a} \otimes A_{1} \oplus I_{b} \otimes B_{1}\right), \ldots,\left(I_{a} \otimes A_{m} \oplus I_{b} \otimes B_{m}\right)\right\}
$$

of order $a n_{1}+b n_{2}=h t$. It can be seen that this family satisfies the conditions of Lemma 11; therefore it makes $\mathrm{OD}\left(h t ; u_{1}, \ldots, u_{m}\right)$.

Now if $A$ and $B$ are symmetric, then $A_{i}$ 's and $B_{i}$ 's, $1 \leq i \leq$ $m$, are all symmetric. Since

$$
((A \otimes B) \oplus(C \otimes D))^{T}=\left(A^{T} \otimes B^{T}\right) \oplus\left(C^{T} \otimes D^{T}\right),
$$

set $S$ consists of $m$ symmetric matrices of order $h t$ satisfying the conditions of Lemma 11, and so they generate symmetric $\mathrm{OD}\left(h t ; u_{1}, \ldots, u_{m}\right)$.

Theorem 14 (Wallis and Whiteman [7]). Let $q$ be a prime power. Then there is circulant $W\left(q^{2}+q+1, q^{2}\right)$.

Corollary 15 (see $[1,8])$. Suppose that $q$ is a prime power and $c$ is any positive integer. Then there is circulant $W\left(c\left(q^{2}+q+\right.\right.$ 1), $\left.q^{2}\right)$.

Proof. Let $c$ be a fixed positive integer. From Theorem 14, we know that there exists a circulant $W\left(q^{2}+q+1, q^{2}\right)$. Suppose that the first row of this matrix is $\left(a_{1}, a_{2}, \ldots, a_{q^{2}+q+1}\right)$. Let

$$
\phi(x)=\sum_{i=1}^{q^{2}+q+1} a_{i} x^{i}
$$

Thus, $\phi(\xi) \phi\left(\xi^{-1}\right)=q^{2}$, where $\xi$ is a primitive root of unity and $\xi^{q^{2}+q+1}=1$. For $1 \leq j \leq c\left(q^{2}+q+1\right)$ define

$$
b_{j}:= \begin{cases}a_{\lceil j / c\rceil} & j \equiv 1(\bmod c) \\ 0 & \text { otherwise }\end{cases}
$$

where $\lceil x\rceil$ is the smallest integer greater than or equal to $x$. We show that if $W=\operatorname{circ}\left(b_{1}, b_{2}, \ldots, b_{c\left(q^{2}+q+1\right)}\right)$, then $W$ is $W\left(c\left(q^{2}+\right.\right.$ $\left.q+1), q^{2}\right)$. To see this, let

$$
\psi(y)=\sum_{j=1}^{c\left(q^{2}+q+1\right)} b_{j} y^{j}
$$

Thus we have

$$
\psi(y)=\sum_{i=1}^{q^{2}+q+1} a_{i} y^{c(i-1)+1}=y^{1-c} \sum_{i=1}^{q^{2}+q+1} a_{i} y^{c i}
$$

Since $\phi(\xi) \phi\left(\xi^{-1}\right)=q^{2}$, for all $\xi$ such that $\xi^{q^{2}+q+1}=$ $1, \psi(\xi) \psi\left(\xi^{-1}\right)=q^{2}$, for all $\xi$ such that $\xi^{q^{2}+q+1}=1$. Applying the finite Parseval relation

$$
\sum_{i=1}^{c\left(q^{2}+q+1\right)} b_{i} b_{i+r}=\frac{1}{c\left(q^{2}+q+1\right)} \sum_{j=1}^{c\left(q^{2}+q+1\right)}\left|\psi\left(\xi^{j}\right)\right|^{2} \xi^{j r},
$$

where $i+r-1$ is reduced modulo $c\left(q^{2}+q+1\right)$, for $r=0$ gives

$$
\sum_{i=1}^{c\left(q^{2}+q+1\right)} b_{i}^{2}=\frac{1}{c\left(q^{2}+q+1\right)}\left(c\left(q^{2}+q+1\right) q^{2}\right)=q^{2} .
$$

And, for $1 \leq r \leq c\left(q^{2}+q+1\right)-1, \sum_{i=1}^{c\left(q^{2}+q+1\right)} b_{i} b_{i+r}=0$. Therefore, $W$ is circulant $W\left(c\left(q^{2}+q+1\right), q^{2}\right)$. 
The next lemma shows how to make a symmetric OD to be used for Theorem 21 .

Lemma 16. Let $k$ be a positive integer. Then there exists symmetric $O D\left(2^{k} ; 1_{(k)}\right)$.

Proof. Define $A_{1}=\bigotimes_{m=1}^{k} P$ and, for $2 \leq n \leq k, A_{n}=$ $\bigotimes_{m=1}^{n-2} I \otimes Q \bigotimes_{m=n}^{k} P$, where

$$
\begin{gathered}
P=\left[\begin{array}{ll}
0 & 1 \\
1 & 0
\end{array}\right], \\
Q=\left[\begin{array}{cc}
1 & 0 \\
0 & -1
\end{array}\right], \\
I=\left[\begin{array}{ll}
1 & 0 \\
0 & 1
\end{array}\right] .
\end{gathered}
$$

It can be directly verified that the family $\left\{A_{1}, \ldots, A_{k}\right\}$ of order $2^{k}$ satisfies the conditions of Lemma 11 , and therefore it makes symmetric $\operatorname{OD}\left(2^{k} ; 1_{(k)}\right)$. Note that $P, Q$, and $I$ are symmetric.

Theorem 17 (Robinson [9]). All $O D\left(2^{t} ; 1,1, a, b, c\right)$ exist, where $t \geq 3$ and $a+b+c=2^{t}-2$.

We prove the following well known lemma by giving a proof which is different from the proof in [1, Lemma 7.27].

Lemma 18. For any sequence $\left(k_{1}, k_{2}, k_{3}, k_{4}\right)$ of positive integers, there is a positive integer $d$ such that there is skewsymmetric $O D\left(2^{d} ; k_{1}, k_{2}, k_{3}, k_{4}\right)$.

Proof. Let $t_{1}$ and $t_{2}$ be the smallest positive integers such that $1+k_{1}+k_{2} \leq 2^{t_{1}}$ and $1+k_{3}+k_{4} \leq 2^{t_{2}}$. By Theorem 17, there are $A=\mathrm{OD}\left(2^{t_{1}} ; 1, k_{1}, k_{2}\right)$ and $B=\mathrm{OD}\left(2^{t_{2}} ; 1, k_{3}, k_{4}\right)$. Without loss of generality, assume that $\left\{I_{2^{t_{1}}}, A_{1}, A_{2}\right\}$ and $\left\{I_{2^{t_{2}}}, B_{1}, B_{2}\right\}$ are two families corresponding to $A$ and $B$ satisfying the conditions of Lemma 11 . Let $P$ and $Q$ be the same matrices as in the proof of Lemma 16. It can be directly verified that the family

$$
\begin{aligned}
& \left\{I_{2^{t_{2}}} \otimes A_{1} \otimes P, I_{2^{t_{2}}} \otimes A_{2} \otimes P, B_{1} \otimes I_{2^{t_{1}}} \otimes Q, B_{2} \otimes I_{2^{t_{1}}}\right. \\
& \quad \otimes Q\}
\end{aligned}
$$

of four skew-symmetric matrices satisfies all conditions of Lemma 11, and so it makes skew-symmetric $\mathrm{OD}\left(2^{t_{1}+t_{2}+1} ; k_{1}, k_{2}, k_{3}, k_{4}\right)$.

Corollary 19 (see [1]). Given any sequence $\left(k_{1}, k_{2}, k_{3}, k_{4}\right)$ of positive integers, there exists a positive integer $d$ such that there is $\mathrm{OD}\left(2^{d} ; 1, k_{1}, k_{2}, k_{3}, k_{4}\right)$. known.

The following theorem, due to Geramita and Seberry, is

Theorem 20 (Geramita and Seberry [1, Theorem 7.14]). Suppose that $k$ is a square. Then there is an integer $N=N(k)$ such that, for each $n \geq N$, there is $W(n, k)$.
We use a slightly different method to the proof of Theorem 20 to give a proof of the following improved result.

Theorem 21. Suppose that $k$ is a square. Then there is an integer $N=N(k)$ such that, for each $n \geq N$, there is symmetric $W(n, k)$.

Proof. Assume that $k=\prod_{i=1}^{m} q_{i}^{2}$, where $q_{i}$ is either 1 or a prime power. By Theorem 14, for each $i$ there exists circulant $W_{i}=$ $W\left(q_{i}^{2}+q_{i}+1, q_{i}^{2}\right)$. Let

$$
W=\bigotimes_{i=1}^{m} W_{i} R_{i},
$$

where $R_{i}$ is the back-diagonal matrix of order $q_{i}^{2}+q_{i}+1$. It can be seen that $W$ is symmetric $W\left(\prod_{i=1}^{m}\left(q_{i}^{2}+q_{i}+1\right), \prod_{i=1}^{m} q_{i}^{2}\right)$.

Thus, there is an odd number $t=\prod_{i=1}^{m}\left(q_{i}^{2}+q_{i}+1\right)$ such that there is symmetric $W(t, k)$. Moreover, from Lemma 16 , there exists symmetric $\operatorname{OD}\left(2^{k} ; 1_{(k)}\right)$, and so there is symmetric $W\left(2^{k}, k\right)$. Now since $t$ is odd, $\operatorname{gcd}\left(2^{k}, t\right)=1$. Lemma 13 implies that there is a positive integer $N=N(k)$ such that, for each $n \geq N$, there exists symmetric $W(n, k)$.

We prove the following theorem by a slightly different method to the proof that first was given by Eades $[1,8$, Theorem 7.15].

Theorem 22. Suppose that $k=k_{1}^{2}+k_{2}^{2}$, where $k_{1}$ and $k_{2}$ are two nonzero integers. Then there is an integer $N=N(k)$ such that, for each $n \geq N$, there is $O D\left(2 n ; k_{1}^{2}, k_{2}^{2}\right)$.

Proof. For $j=1,2$, let $k_{j}^{2}=\prod_{i=1}^{m} q_{i j}^{2}$, where $q_{i j}$ is either 1 or a prime power. For each $i, 1 \leq i \leq m$, let $b_{i}=\operatorname{lcm}\left\{q_{i 1}^{2}+q_{i 1}+\right.$ $\left.1, q_{i 2}^{2}+q_{i 2}+1\right\}$. From Corollary 15 , for each $j, j=1,2$, and each $i, 1 \leq i \leq m$, there exists circulant $W_{i j}=W\left(b_{i}, q_{i j}^{2}\right)$. It can be seen that the following $2 q \times 2 q$ matrix is $\mathrm{OD}\left(2 q ; k_{1}^{2}, k_{2}^{2}\right)$,

$$
\left[\begin{array}{cc}
x \bigotimes_{i=1}^{m} W_{i 1} R_{i} & y \bigotimes_{i=1}^{m} W_{i 2} \\
y \bigotimes_{i=1}^{m} W_{i 2} & -x \bigotimes_{i=1}^{m} W_{i 1} R_{i}
\end{array}\right],
$$

where $R_{i}$ is the back-diagonal matrix of order $b_{i}$ and $q=\prod_{i=1}^{m} b_{i}$ is an odd number. From Theorem 17, one can choose the smallest positive integer $k$ such that there is $\mathrm{OD}\left(2^{k} ; k_{1}^{2}, k_{2}^{2}\right)$. Since $\operatorname{gcd}\left(2 q, 2^{k}\right)=2$, Lemma 13 implies that there is an integer $N=N(k)$ such that, for each $n \geq N$, there is $\mathrm{OD}\left(2 n ; k_{1}^{2}, k_{2}^{2}\right)$.

Using the methodology in the proof of Theorem 22, the asymptotic bounds for the following two corollaries given by Eades [8] are improved.

Corollary 23. Suppose that $k$ is the sum of two nonzero integer squares. Then there is an integer $N=N(k)$ such that, for each $n \geq N$, there is $W(2 n, k)$.

Proof. Let $k=k_{1}^{2}+k_{2}^{2}$, where $k_{1}$ and $k_{2}$ are integers. From Theorem 22, there is an integer $N=N(k)$ such that, for any $n \geq N$, there is $\operatorname{OD}\left(2 n ; k_{1}^{2}, k_{2}^{2}\right)$, and so there is $W(2 n, k)$. 
Corollary 24. Suppose that $d$ is an integer square. Then there exists an integer $N=N(d)$ such that, for each $n \geq N$, there is skew-symmetric $W(2 n, d)$.

Proof. Suppose that $d=a^{2}$. Let $k_{1}=1$ and $k_{2}=a$. By Theorem 22, there exists an integer $N=N(d)$ such that, for each $n \geq N$, there is $\operatorname{OD}(2 n ; 1, d)$, and so there is skewsymmetric $W(2 n, d)$.

We now use a different method to show Theorem 25 shown by Eades [1, 8, Theorem 7.17] to improve the bounds $(N)$ for the asymptotic existence of ODs of order $4 n$, and consequently we prove Corollaries 26, 27, and 28.

Theorem 25. Suppose that $k=k_{1}^{2}+k_{2}^{2}+k_{3}^{2}+k_{4}^{2}$, where $k_{1}, k_{2}, k_{3}$, and $k_{4}$ are nonzero integers. Then there is an integer $N=N(k)$ such that, for each $n \geq N$, there is $O D\left(4 n ; k_{1}^{2}, k_{2}^{2}, k_{3}^{2}, k_{4}^{2}\right)$.

Proof. Assume that $k=k_{1}^{2}+k_{2}^{2}+k_{3}^{2}+k_{4}^{2}$ and $k_{1}, k_{2}, k_{3}$, and $k_{4}$ are nonzero integers. Let $k_{j}^{2}=\prod_{i=1}^{m} q_{i j}^{2}$, where $q_{i j}$ is either 1 or a prime power. For each $i, 1 \leq i \leq m$, let $b_{i}=\operatorname{lcm}\left\{q_{i j}^{2}+q_{i j}+1 ; j=\right.$ $1,2,3,4\}$. From Corollary 15 , for each $j, 1 \leq j \leq 4$, and each $i, 1 \leq i \leq m$, there exists circulant $W_{i j}=W\left(b_{i}, q_{i j}^{2}\right)$. Putting

$$
\begin{aligned}
& A=\bigotimes_{i=1}^{m} W_{i 1} R_{i}, \\
& B=\bigotimes_{i=1}^{m} W_{i 2}, \\
& C=\bigotimes_{i=1}^{m} W_{i 3}, \\
& D=\bigotimes_{i=1}^{m} W_{i 4},
\end{aligned}
$$

in the following array (Goethals and Seidel [10]) gives $\mathrm{OD}\left(4 q ; k_{1}^{2}, k_{2}^{2}, k_{3}^{2}, k_{4}^{2}\right)$ :

$$
\left[\begin{array}{cccc}
x A & y B & z C & u D \\
-y B & x A & u D^{T} & -z C^{T} \\
-z C & -u D^{T} & x A & y B^{T} \\
-u D & z C^{T} & -y B^{T} & x A
\end{array}\right]
$$

where $q=\prod_{i=1}^{m} b_{i}$ which is an odd number and $R_{i}$ is the backdiagonal matrix of order $b_{i}$.

By Lemma 18 , there is $\operatorname{OD}\left(2^{d} ; k_{1}^{2}, k_{2}^{2}, k_{3}^{2}, k_{4}^{2}\right)$ for some suitable integer $d \geq 2$. Since, for $d \geq 2, \operatorname{gcd}\left(4 q, 2^{d}\right)=4$, Lemma 13 implies that there is an integer $N=N(k)$ such that, for each $n \geq N$, there is $\operatorname{OD}\left(4 n ; k_{1}^{2}, k_{2}^{2}, k_{3}^{2}, k_{4}^{2}\right)$. Note that if some of $k_{i}$ 's are zero, then we consider the zero matrices.

Corollary 26. Suppose that $d$ is any positive integer. Then there is an integer $N=N(d)$ such that, for each $n \geq N$, there is $W(4 n, d)$.
Proof. It is a well known theorem of Lagrange [11] that every positive integer can be written in the sum of four integer squares. Let $d=k_{1}^{2}+k_{2}^{2}+k_{3}^{2}+k_{4}^{2}$. From Theorem 25 , there is an integer $N=N(k)$ such that, for each $n \geq N$, there is $\mathrm{OD}\left(4 n ; k_{1}^{2}, k_{2}^{2}, k_{3}^{2}, k_{4}^{2}\right)$, and therefore there is $W(4 n, d)$.

Corollary 27. Suppose that $d$ is the sum of three integer squares. Then there exists an integer $N=N(d)$ such that, for each $n \geq N$, there is skew-symmetric $W(4 n, d)$.

Proof. Consider $d=a^{2}+b^{2}+c^{2}$, for some integers $a, b$, and $c$. Substituting $k_{1}=a, k_{2}=b, k_{3}=c$, and $k_{4}=1$ in Theorem 25 gives the result. Note that the existence of $\operatorname{OD}(n ; 1, h)$ is equivalent to existence of skew-symmetric $W(n, h)$.

Corollary 28. Suppose that $d$ is any positive integer. Then there exists an integer $N=N(d)$ such that, for each $n \geq N$, there is skew-symmetric $W(8 n, d)$.

Proof. By Lagrange's theorem [12], one can write $d=k_{1}^{2}+k_{2}^{2}+$ $k_{3}^{2}+k_{4}^{2}$, where $k_{i}$ 's are nonnegative integers. Let $A, B, C$, and $D$ be the same matrices as in Theorem 25. It can be seen that the following matrix gives $\operatorname{OD}\left(8 q ; 1, k_{1}^{2}, k_{2}^{2}, k_{3}^{2}, k_{4}^{2}\right)$, where $q$ is obtained as in Theorem 25, and is an odd number:

$$
\left[\begin{array}{cccc|cccc}
x A & y B & z C & u D & w I_{q} & 0 & 0 & 0 \\
-y B & x A & u D^{T} & -z C^{T} & 0 & w I_{q} & 0 & 0 \\
-z C & -u D^{T} & x A & y B^{T} & 0 & 0 & w I_{q} & 0 \\
-u D & z C^{T} & -y B^{T} & x A & 0 & 0 & 0 & w I_{q} \\
\hline w I_{q} & 0 & 0 & 0 & -x A & y B^{T} & z C^{T} & u D^{T} \\
0 & w I_{q} & 0 & 0 & -y B^{T} & -x A & u D & -z C \\
0 & 0 & w I_{q} & 0 & -z C^{T} & -u D & -x A & y B \\
0 & 0 & 0 & w I_{q} & -u D^{T} & z C & -y B & -x A
\end{array}\right] .
$$

From Corollary 19 , there is $\mathrm{OD}\left(2^{d} ; 1, k_{1}^{2}, k_{2}^{2}, k_{3}^{2}, k_{4}^{2}\right)$ for some suitable integer $d \geq 3$. Since, for $d \geq 3, \operatorname{gcd}\left(8 q, 2^{d}\right)=8$, Lemma 13 implies that there is an integer $N=N(d)$ such that, for any $n \geq N$, there is $\operatorname{OD}\left(8 n ; 1, k_{1}^{2}, k_{2}^{2}, k_{3}^{2}, k_{4}^{2}\right)$, and so there is skew-symmetric $W(8 n, d)$.

Example 29. Suppose that $k=92$. Let $k_{1}=2, k_{2}=4, k_{3}=6$, and $k_{4}=6$ in Theorem 25. Also, let $q_{11}=2, q_{21}=1, q_{12}=$ $4, q_{22}=1, q_{13}=2, q_{23}=3, q_{14}=2$, and $q_{24}=3$. Then $b_{1}=\operatorname{lcm}\{7,21,7,7\}=21$, and $b_{2}=\operatorname{lcm}\{3,3,13,13\}=39$. By Theorem 25, there is

$$
\operatorname{OD}\left(4 \cdot 21 \cdot 39 ; 2^{2}, 4^{2}, 6^{2}, 6^{2}\right)
$$

From Lemma 18, there is $\mathrm{OD}\left(2^{13} ; 2^{2}, 4^{2}, 6^{2}, 6^{2}\right)$. By Lemma 13, since $h=\operatorname{gcd}\left(4 \cdot 21 \cdot 39,2^{13}\right)=4$, we have $N(92) \leq 2^{11} \cdot 3^{2} \cdot 7 \cdot 13$, and so, for each $n \geq N(92)$, there are $W(4 n, 92)$ and skewsymmetric $W(8 n, 92)$.

\section{Competing Interests}

The author declares that there is no conflict of interests regarding the publication of this paper. 


\section{Acknowledgments}

The paper constitutes Chapter 3 of the author's Ph.D. thesis written under the direction of Professor Hadi Kharaghani at the University of Lethbridge. The author would like to thank Professor Hadi Kharaghani for introducing the problem and his very useful guidance toward solving the problem and also Professor Rob Craigen for his time and great help.

\section{References}

[1] A. V. Geramita and J. Seberry, Orthogonal Designs: Quadratic Forms and Hadamard Matrices, vol. 45 of Lecture Notes in Pure and Applied Mathematics, Marcel Dekker, New York, NY, USA, 1979.

[2] E. Ghaderpour, Asymptotic existence of orthogonal designs [Ph.D. thesis], University of Lethbridge, Lethbridge, Canada, ProQuest LLC, Ann Arbor, Mich, USA, 2013.

[3] R. Craigen, Constructions for orthogonal matrices [Ph.D. thesis], University of Waterloo, Waterloo, Canada, 1991.

[4] J. P. Serre, A Course in Arithmetic, Graduate Texts in Mathematics, No. 7, Springer, New York, NY, USA, 1973, Translated from the French.

[5] D. B. Shapiro, Similarities, quadratic forms, and Clifford algebras [Ph.D. thesis], ProQuest, Ann Arbor, Mich, USA; University of California, Berkeley, Berkeley, Calif, USA, 1974.

[6] J. J. Sylvester, “Thoughts on inverse orthogonal matrices, simultaneous sign- successions, and tessellated pavements in two or more colors, with applications to Newton's rule, ornamental tilework, and the theory of numbers," Philosophical Magazine, vol. 34, pp. 461-475, 1867.

[7] J. S. Wallis and A. L. Whiteman, "Some results on weighing matrices," Bulletin of the Australian Mathematical Society, vol. 12, no. 3, pp. 433-447, 1975.

[8] P. Eades, "Some asymptotic existence results for orthogonal designs," Ars Combinatoria, vol. 1, no. 1, pp. 109-118, 1976.

[9] P. J. Robinson, "Using product designs to construct orthogonal designs," Bulletin of the Australian Mathematical Society, vol. 16, no. 2, pp. 297-305, 1977.

[10] J.-M. Goethals and J. J. Seidel, "Orthogonal matrices with zero diagonal," Canadian Journal of Mathematics, vol. 19, pp. 10011010, 1967.

[11] K. Ireland and M. I. Rosen, Elements of Number Theory: Including an Introduction to Equations over Finite Fields, Bogden \& Quigley, Tarrytown, NY, USA, 1972.

[12] I. M. Vinogradov, Elements of Number Theory, Dover Publications, New York, NY, USA, 1954, Translated by: S. Kravetz. 


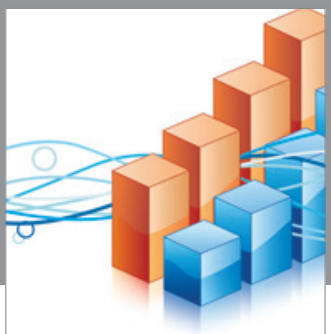

Advances in

Operations Research

vatem alat4

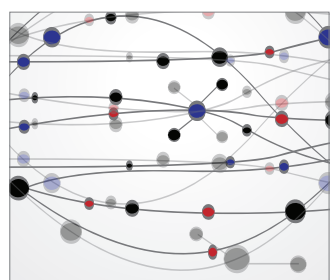

\section{The Scientific} World Journal
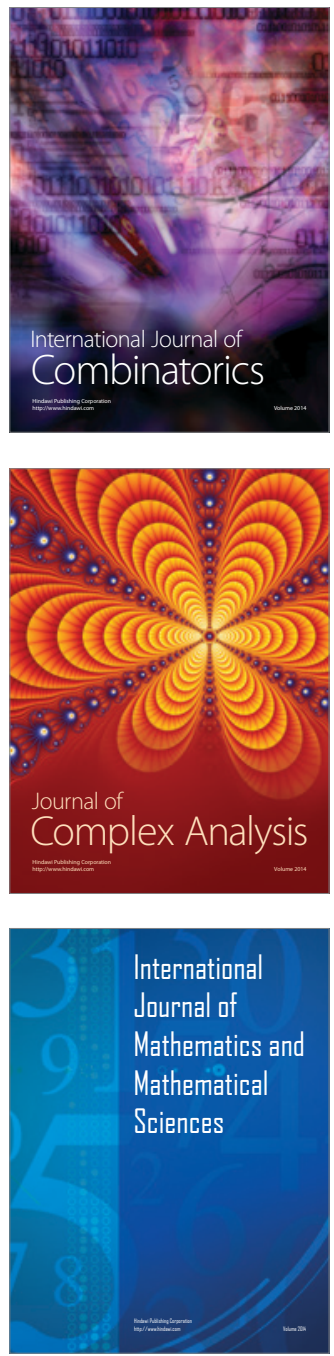
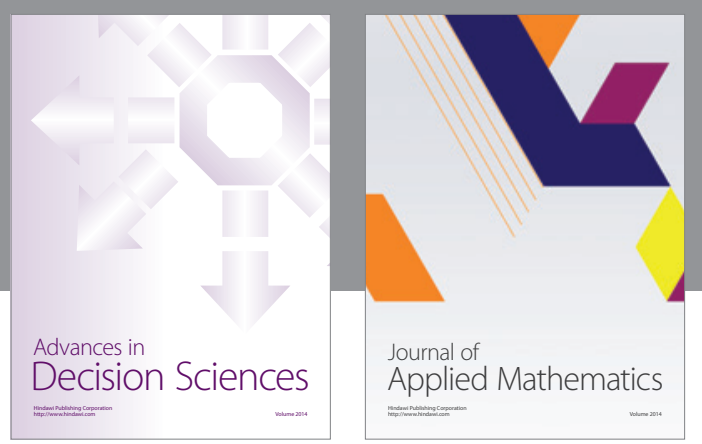

Algebra

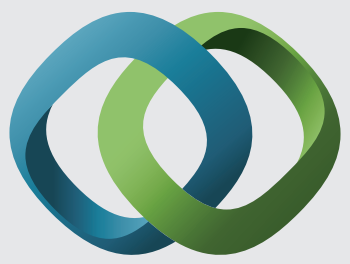

\section{Hindawi}

Submit your manuscripts at

http://www.hindawi.com
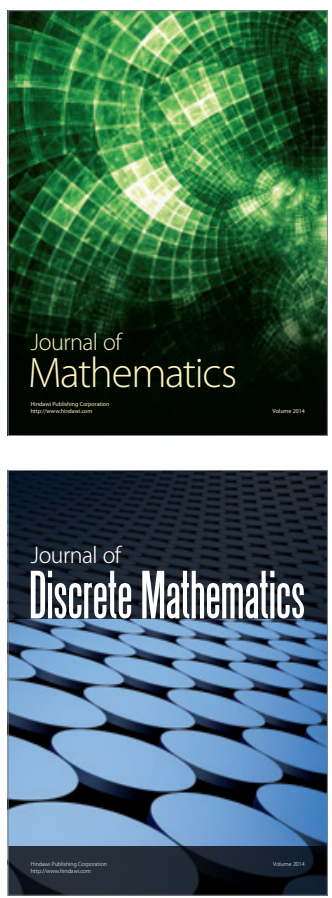

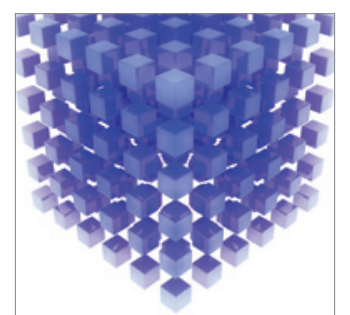

Mathematical Problems in Engineering
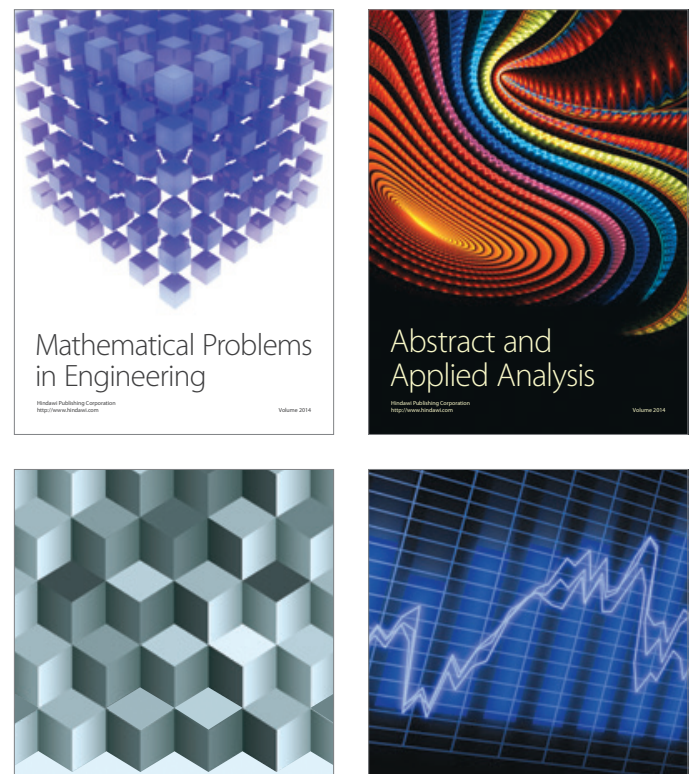

Journal of

Function Spaces

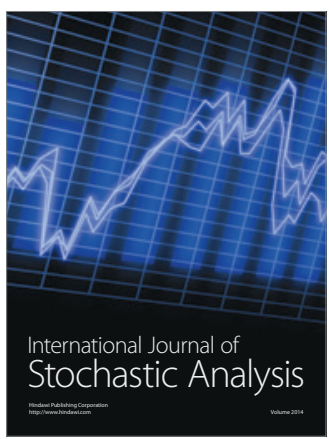

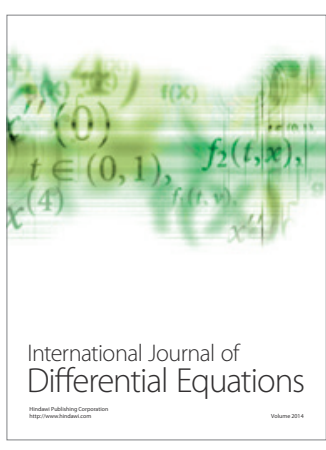
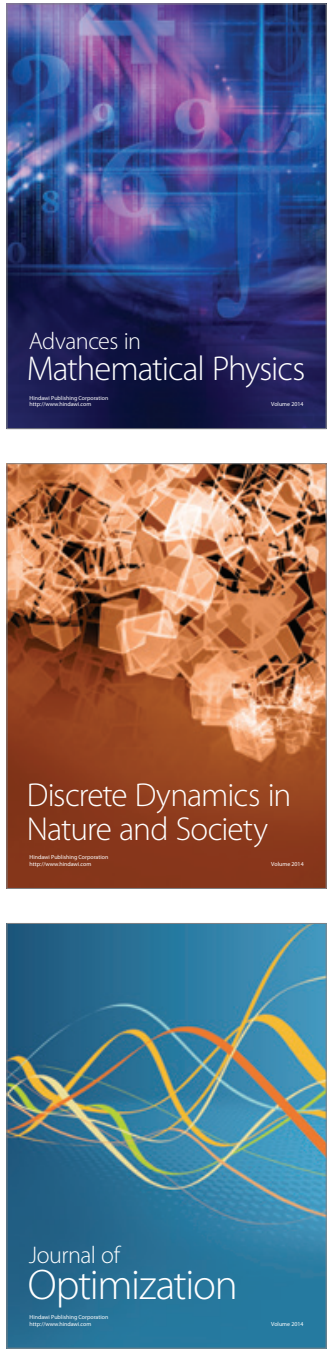